\title{
ADAPTIVE OPTICS OBSERVATIONS OF VEGA: EIGHT DETECTED SOURCES AND UPPER LIMITS TO PLANETARY-MASS COMPANIONS
}

\author{
Stanimir A. Metchev, Lynne A. Hillenbrand, and Russel J. White \\ California Institute of Technology, Department of Astronomy, MC 105-24, Pasadena, CA 91125; \\ metchev@astro.caltech.edu,lah@astro.caltech.edu,rjw@astro.caltech.edu \\ Received 2002 July 26; accepted 2002 September 17
}

\begin{abstract}
From adaptive optics observations with the Palomar $5 \mathrm{~m}$ telescope we place upper limits on the masses of any planetary companions located between $\sim 30$ and 230 AU away from Vega, where our data are sensitive to depths ranging from $H=12.5-19.0$ mag fainter than Vega itself. Our observations cover a plus-shaped area with two $25^{\prime \prime} \times 57^{\prime \prime}$ elements, excluding $7^{\prime \prime} \times 7^{\prime \prime}$ centered on the star. We have identified two double and four single point sources. These projected companions are 14.9-18.9 mag fainter than Vega and, if physically associated, would have masses ranging from 4 to $35 M_{\mathrm{J}}$ and orbital radii of 170-260 AU. Recent simulations of dusty rings around Vega predict the presence of a perturbing body with a mass of less than 2-3 $M_{\mathrm{J}}$ and an orbital radius of $\sim 40-100 \mathrm{AU}$, and more massive ( $\lesssim 10 M_{\mathrm{J}}$ ) planets cannot be excluded. None of the detected objects are this predicted planet. Based on a color-magnitude, spectroscopic, and proper motion analysis, all objects are consistent with being background sources. Given the glare of Vega, a $2 M_{\mathrm{J}}$ object near the expected orbital radii would not have been visible at the $5 \sigma$ level in our data, though any brown dwarf with mass greater than $10 M_{\mathrm{J}}$ could have been seen at a separation greater than $80 \mathrm{AU}$.
\end{abstract}

Subject headings: instrumentation: adaptive optics — instrumentation: interferometers stars: imaging — stars: individual (Vega) — stars: low-mass, brown dwarfs

\section{INTRODUCTION}

The A $0 \mathrm{~V}$ star Vega has been famous since the early days of data return from IRAS as a young main-sequence star surrounded by dust (Aumann et al. 1984). Its age (270-380 Myr; Song et al. 2001) combined with the large fractional excess luminosity at infrared wavelengths $\left(L_{\text {excess }} / L_{*} \approx\right.$ $10^{-5}$ or $M_{\text {dust }} \approx \frac{1}{2} M_{\text {moon }}$; Backman \& Paresce 1993) imply that dust is being generated at the current epoch by either grinding collisions between larger rocky bodies, aka planetesimals (Harper, Loewenstein, \& Davidson 1984; Weissman 1984; Zuckerman \& Becklin 1993), or in cometary ejecta (Beust et al. 1989, 1990, and references therein). If the dust is not continuously regenerated it will be depleted by a combination of Poynting-Robertson drag and radiation pressure on a timescale much shorter than the age of Vega. Discovery of the infrared excess around Vega and other main-sequence stars too old to possess the so-called primordial dust and gas disks that are commonly found around 1-10 Myr old stars, led to coining of the term "debris disk." Searches for other examples of "the Vega phenomenon" have led to cataloging of mere tens of objects (see, e.g., Mannings \& Barlow 1998; Silverstone 2000), mostly earlytype stars whose dust was detectable with IRAS or ISO, or observable from the ground with mid-infrared instrumentation on large telescopes.

The mid- and far-infrared $(25-850 \mu \mathrm{m})$ emission from Vega is extended over tens of arcseconds (Aumann et al. 1984; Harvey, Wilking, \& Joy 1984; Zuckerman \& Becklin 1993; Heinrichsen, Walker, \& Klaas 1998; Holland et al. 1998). Aperture synthesis imaging at $1.3 \mathrm{~mm}$ (Koerner, Sargent, \& Ostroff 2001; Wilner et al. 2002) resolved several dust clumps located $\sim 8^{\prime \prime}-14^{\prime \prime}$ from the central source $(60$ $110 \mathrm{AU}$, assuming the Hipparcos parallax of 128.9 mas). One interpretation is that these clumps trace the densest portions of the already inferred face-on circumstellar ring
(Dent et al. 2000). Additional support for a ring interpretation comes from Vega's spectral energy distribution, which is close to photospheric at shorter wavelengths ( $\$ 20 \mu \mathrm{m}$; Heinrichsen et al. 1998), and suggests an inner gap in the density distribution, which may or may not be entirely devoid of hot dust. At $11.6 \mu \mathrm{m}$ extensions larger than 0 ".25 are ruled out by the imaging of Kuchner, Brown, \& Koresko (1998). Interferometric work by Ciardi et al. (2001), however, did suggest extended emission at $2.2 \mu \mathrm{m}$.

Observations of structure in the circumstellar dust around Vega have spawned detailed models for a planetary perturber (Gorkavyi \& Taidakova 2001; Wilner et al. 2002). Resonance trapping and gravitational scattering induced by a body of mass 2-3 $M_{\mathrm{J}}$ are consistent with the Holland et al. (1998) map, and with the interferometric observations of Koerner et al. (2001) and Wilner et al. (2002). Because of degeneracies in dynamic models (e.g., Wilner et al. 2002), more massive planets $\left(\sim 10 M_{\mathrm{J}}\right)$ also cannot be ruled out. Modeling to date assumes a face-on orientation of the presumed dust disk or ring. Evidence for this geometry comes both from a ring-shaped (e.g., Heinrichsen et al. 1998) albeit clumpy (Koerner et al. 2001; Wilner et al. 2002) dust distribution and from detailed analysis of stellar line profiles (assuming parallel disk and stellar rotation axes; Gulliver, Hill, \& Adelman 1994).

Our experiment was designed to search for low-mass companions within $4^{\prime \prime}-30^{\prime \prime}$ of Vega, in part to test the aforementioned planetary perturber predictions. Imaging observations close to this bright source are usually "burned out" in survey data such as POSS or the Two Micron All Sky Survey (2MASS). Ground-based coronagraphic observations (Smith, Fountain, \& Terrile 1992; Kalas \& Jewitt 1996) have also lacked sufficient sensitivity. Except for NICMOS images (Silverstone, Schneider, \& Smith 2002) with sensitivity comparable to ours, high dynamic-range observations have not been previously reported. 


\section{OBSERVATIONS}

Data were obtained with the Palomar adaptive optics (PALAO; Troy et al. 2000; Bloemhof et al. 2000) system in residence at the Palomar $5 \mathrm{~m}$ telescope. PALAO employs PHARO, the Palomar High Angular Resolution Observer (Hayward et al. 2001), a $1024^{2}$ pixel $\mathrm{HgCdTe}$ HAWAII detector with imaging $\left(25^{\prime \prime}\right.$ or $40^{\prime \prime}$ field of view) and spectroscopic $(R=1500-2500)$ capabilities. Broad- and narrowband filters throughout the $J H K$ atmospheric windows are available, as well as a choice of coronagraphic spot sizes and Lyot masks.

Vega was observed on the night of 2002 June 22, with additional follow-up observations obtained on August 28 and 29 (UT), all under photometric sky conditions. The observing strategy was to take deep images in $H$ band to maximize the detection likelihood of faint low-mass objects (see, e.g., Burrows et al. 1997). The point spread function (PSF) was 0."6-0".9 uncorrected at $H$ band and improved to less than 0 ". 1 with adaptive correction. A neutral density filter (1\%) manually placed in front of the wave front sensor (WFS) enabled an AO lock on such a bright object. The AO performance was very good during most of the observing, with Strehl ratios up to $20 \%$ in $H$. We did not employ the coronagraphic mode of PALAO for these observations since scattered light suppression was not sufficient to prevent saturation on the array outside the boundaries of the largest coronagraph $(0.97=$ $12 \lambda / D$ in $H)$ in the shortest possible integration time (1897 ms).

On June 22, a total of 26 minutes on-source integration was obtained with the $25^{\prime \prime}$ field of view in $H$ band at each of four pointings: north, south, east, and west around Vega (hereafter Vega N, S, E, and W fields), with Vega itself located 3".5 off of the imaging field at each positioning of the telescope. Because of field overlaps, $\sim 13 \%$ of the area covered $\left(2210 \operatorname{arcsec}^{2}\right)$ was observed for 52 minutes. Dithering at the $0.25-1$ ".00 level was performed for the on-source frames. More widely dithered sky frames were taken at locations $\sim 2^{\prime}$ farther away from Vega with source-to-sky time split $2: 1$. The integration time for individual exposures was 10.9 s. For the eastern field in which several objects were noticed in real time, we also obtained $J, H$, and $K_{s}$ data with 2.5 minutes total on-source integration time taken as five separate frames, with Vega offset $22^{\prime \prime}-28^{\prime \prime}$ to the west. The air-mass range was $1.03-1.30$ for the entire observing sequence.

Photometric calibration was achieved via immediate observation of 2MASS 183726.28+385210.1 (GSC 0310500679, a G8 V star) located 7.7 from Vega with 2MASS magnitudes $K_{s}=8.296 \pm 0.033, H=8.365 \pm 0.022$, and $J=8.745 \pm 0.028$. This source, although not a photometric standard, is sufficient as a local calibrator and was observed at air mass 1.35. Two other much fainter 2MASS sources are also present in the image.

During the second epoch observations, resolution $R=1500$ and $2400 \mathrm{~K}$-band spectra of the brightest discovered object were obtained (August 28) through a 0"52 slit and a $K$ grism for a total of 100 minutes on-source integration. The object was dithered $10^{\prime \prime}$ along the slit for skysubtraction. Spectra of scattered light from Vega were used as a telluric standard. Short-exposure (5 minutes per filter) dithered $J H K_{s}$ images were taken (August 29) as follow-up to the June 22 data to test for common proper motion with
Vega. The air mass of Vega for the second epoch observations varied between 1.01 and 1.13.

We also observed a binary system (HD 165341) with a well-determined orbit in the Sixth Catalog of Orbits of Visual Binary Stars ${ }^{1}$ in order to determine precisely the platescale and orientation of the PALAO system. We derive for the $25^{\prime \prime}$ field a plate-scale of 0 ".025168 \pm 0 ".000034 pixel $^{-1}$.

\section{DATA PROCESSING}

Our image reduction steps, written in IDL and IRAF, include the standard procedures of flat-fielding, skysubtracting, interpolating/masking bad pixels, and mosaicking the dither pattern. This last step required correcting for image drift (likely caused by change in the direction of the gravity vector in PHARO over the duration of the observing sequence), the rate of which varied between 0.5 and $1 . " 2 \mathrm{hr}^{-1}$.

Image stacks from each of the four deep pointings (June 22) were registered to the first image in the series. For the east field in which several point sources were detected, each image was registered by centroiding on the brightest object. For the north field, centroiding was possible on the bright reflection artifact due to Vega. For the other two fields registration was accomplished by first averaging sets of nine consecutive exposures, extrapolating the position of Vega from the intersection of six scattered light "rays" in the image halo, and combining the 16 registered averages. In this manner, the location of the star could be constrained to within \pm 5.0 pixels $= \pm 0$ " 13 (cf. \pm 0.10 pixels for our mean centroiding precision in the north and east fields). We did attempt cross-correlation techniques for dither pattern correction but these were not as successful as the above procedures. The final step was to orient the images with north up and east to the left. Astrometric calibration was established assuming the plate-scale derived from the binary star observations and by reference to the Hipparcos (J2000.0) coordinates of Vega. Our final image of the Vega vicinity is presented in Figure $1 a$.

Various methods to reduce the large halo from Vega were attempted, including reflections, rotations, and data smoothing. Shown in Figure $1 b$ is a difference image, for which a Gaussian-smoothed $(\sigma=5$ pixels, FWHM $=$ 12 pixels; cf. FWHM $=4$ pixels for the point sources) version of the original image has been subtracted. This procedure effectively removes large-scale gradients. Strong artifacts do remain, however, and contribute to our limited sensitivity to point sources within $\sim 10^{\prime \prime}$ of Vega.

Spectra of the brightest point source were extracted using the APALL task within IRAF. A quadratic polynomial was fitted to all pixels with values greater than $10 \%$ of the peak flux along an aperture. Local background was estimated from a region 0 ".50-1".25 from the aperture center. The extracted spectra were divided by that of the telluric standard (with the $2.166 \mu \mathrm{m} \mathrm{Br} \gamma$ absorption feature interpolated over) to correct for instrumental response and atmospheric transmission. Wavelength calibration was done by fitting a dispersion relation to sky $\mathrm{OH}$ emission lines. Finally, the wavelength-calibrated spectra were co-added.

\footnotetext{
${ }^{1}$ Available at http://ad.usno.navy.mil/wds/orb6.html.
} 




(a)



(b)

FIG. 1.- (a) Composite $H$-band mosaic of the Vega region obtained with PALAO. Eight point sources are detected, five to the east, two to the southeast, and one to the north of Vega. The two close, eastern-most objects are just off the edge of the deep exposure of the Vega E field but have been pasted in from our shallower $J H K_{s}$ images, obtained for photometry purposes, to show their location. Similarly, the double source to the southeast was discovered only in the follow-up shallow $J H K_{s}$ observations. A bright "ghost" reflection of Vega is also visible in the north field. (b) Same image, with a smoothed $(\sigma=5$ pixels) version of itself subtracted, to enhance faint sources in the wings of Vega's halo. 


\section{PHOTOMETRY OF DETECTED SOURCES}

The positions of identified sources are indicated in Figure 1. During the first epoch of observations we detected six point sources, five of which were to the east of Vega. Four of these are single while two are a close (0".6) double, which is 0.7 off the east edge of our deep Vega E image, and was observed only in the short $J H K_{s}$ exposures. The sixth point source is in the north field. During the follow-up observations we detected another double (0".8) source southeast of Vega. Point-spread function (PSF) fitting techniques suggest these are in fact all stellar point sources and not partially resolved galaxies.

We performed photometry using both aperture and PSF techniques. First, we used the IRAF/PHOT task in the short exposures with aperture radii of $10\left(K_{s}\right), 18(H)$, and $32(J)$ pixels $(0 " 50,0.90$, and 1.5 diameters on the sky) chosen to correspond to $2 \times \mathrm{FWHM}$ of the image core and to include the first Airy ring. The mode of the counts in a 30-40 pixel annulus provided local sky, which was critical for subtracting residual scattered light from Vega. For sources 1-6, magnitudes in each of the bands were obtained by comparing the measured aperture flux to that of the 2MASS standard in the same aperture. The magnitudes and positions of sources 7 and 8 were bootstrapped from those of source 1, with its error added in quadrature. Air-mass corrections were applied using extinction coefficients for Palomar as previously determined by L. A. H. (0.114, 0.029, and 0.065 mag per air mass in $J, H$, and $K$, respectively). We also used the PSF, PEAK, and ALLSTAR tasks in IRAF/DAOPHOT for PSF-fitting photometry. PSF fitting worked best at $K_{s}$ band but required a large number of iterations at $H$ and $J$ for convergence in part because the stellar profiles are not diffraction limited. Differences between the aperture and PSF-fitting magnitudes are 0.2-0.3 mag (much larger than the formal errors), and the scatter of the PSF magnitudes is $50 \%$ larger than that of the aperture magnitudes at $J$ and $H$.

Our photometry (Table 1) is from apertures, except for sources 4 and 5, for which we simultaneously fit PSF profiles to each of the components of the double source to determine their magnitude difference. A larger aperture (2".5 diameter to include the PSFs of both sources at all bands) is used to measure a combined flux, and individual magnitudes are obtained from the large-aperture magnitude and the magnitude difference from PSF fitting. The photometry for these two sources is less precise because of a more uneven background.
Repeatability of the photometry from frame to frame was assessed using aperture photometry on the calibration field, which is free of the bright background present in the Vega fields. We find $0.04 \mathrm{mag}$ rms scatter between the five frames. For the shallow $J H K_{s}$ exposures near Vega, frame-to-frame differences are larger because of background variations induced by dithering, which placed Vega closer to the image area for some frames than for others. We have included this scatter in our errors.

We do not include a Strehl term in our calibration as the implied corrections were larger than the uncorrected frameto-frame scatter. The Strehl ratio changed from $\sim 15 \%$ in the deep $H$ exposures to $2 \%-3 \%$ in the subsequent shallow ones, but was relatively stable between the short exposures of the object and the calibration fields.

\section{ANALYSIS}

\subsection{Sensitivity Limits}

In the absence of the bright glare from Vega, our deep observations should nominally detect point sources at signal-to-noise-ratio $\mathrm{S} / \mathrm{N}=5$ to $H=20.8$ (21.2, for $13 \%$ of the image), while the shorter $J H K_{s}$ exposures should reach $J=20.8, H=20.1$, and $K_{s}=18.9$. However, the star adds substantial scattered light background and makes pointsource detection a function of position with respect to Vega.

We have assessed our $H$-band detection limits using artificial star experiments, both in the direct image mosaic and in the halo-subtracted image. IRAF/PSF was used to fit the two brightest single objects in the processed Vega E image, and artificial stars were added to the same image with ADDSTAR. A single experiment consisted of adding sources of constant magnitude at $1^{\prime \prime}$ intervals along nine radial (originating from Vega) directions, offset by $15^{\circ}$ from each other. We observed the minimum separation from Vega at which a source would be considered "detected " by eye: at $\mathrm{S} / \mathrm{N} \gtrsim 5$ according to formal $\mathrm{S} / \mathrm{N}$ calculations assuming Gaussian noise statistics. Since the primary source of noise (scattered light from Vega) does not behave in a Gaussian manner, however, the $\mathrm{S} / \mathrm{N}$ statistic does not carry the correct information about the significance of a detection and is only used as an approximate measure of the local contrast.

The experiments were repeated at $0.5 \mathrm{mag}$ steps. For a given radial distance, there are thus up to nine independent measurements of the limiting magnitude (fewer for larger distances, where some artificial sources fall beyond the array), as shown in Figure 2. Our average sensitivity ranges

TABLE 1

Near-Infrared Point Sources in the Vicinity of Vega

\begin{tabular}{|c|c|c|c|c|c|c|c|c|}
\hline ID & $\begin{array}{c}\text { R.A. } \\
\text { (J2000.0) }\end{array}$ & $\begin{array}{c}\text { Decl. } \\
(\mathrm{J} 2000.0)\end{array}$ & $\begin{array}{c}J \\
(\mathrm{mag})\end{array}$ & $\begin{array}{c}H \\
(\mathrm{mag})\end{array}$ & $\begin{array}{c}K_{s} \\
(\mathrm{mag})\end{array}$ & $\begin{array}{c}\text { Separation from Vega } \\
(\operatorname{arcsec})\end{array}$ & $\begin{array}{l}\text { P.A. } \\
\text { (deg) }\end{array}$ & $\begin{array}{l}\text { Mass If Associated } \\
\left(M_{\mathrm{J}}\right)\end{array}$ \\
\hline $1 \ldots \ldots \ldots$ & 183658.19 & +384656.2 & $15.64 \pm 0.07$ & $14.78 \pm 0.05$ & $14.53 \pm 0.06$ & $22.26 \pm 0.03$ & $103.4 \pm 0.1$ & $13-35$ \\
\hline $3 \ldots \ldots \ldots$ & 183658.70 & +384658.4 & $>19.3 \pm 0.1$ & $18.92 \pm 0.12$ & $18.23 \pm 0.12$ & $27.70 \pm 0.03$ & $96.0 \pm 0.1$ & $4-18$ \\
\hline $4 \ldots \ldots \ldots$ & 183659.35 & +384705.5 & $17.15 \pm 0.13$ & $16.25 \pm 0.14$ & $15.98 \pm 0.12$ & $29.41 \pm 0.05$ & $86.2 \pm 0.1$ & $8-27$ \\
\hline $5 \ldots \ldots \ldots$ & 183659.39 & +384705.7 & $16.76 \pm 0.20$ & $16.29 \pm 0.16$ & $16.27 \pm 0.12$ & $29.93 \pm 0.05$ & $85.8 \pm 0.1$ & $8-27$ \\
\hline $6 \ldots \ldots \ldots$ & 183655.36 & +384725.9 & $\ldots$ & $17.43 \pm 0.07$ & $\ldots$ & $27.05 \pm 0.05$ & $335.0 \pm 0.1$ & $6-22$ \\
\hline
\end{tabular}

Note.--Units of right ascension are hours, minutes, and seconds, and units of declination are degrees, arcminutes, and arcseconds.

a Minimum value interpolated from the Burrows et al. 2001 models for $300 \mathrm{Myr}$; maximum value from the Chabrier et al. 2000 models for 500 Myr. 




FIG. 2.- $H$-band sensitivity of our deep images to faint objects as a function of radial distance from Vega analyzed for the case of the east field. Solid points represent individual measurements of the limiting magnitude at different position angles and angular separations from Vega (a slight offset along the abscissa has been applied for clarity). The solid line delineates the azimuthal average as a function of separation. Numbered stars indicate detected point sources. Horizontal arrows indicate the corresponding planetary mass at a given $H$ magnitude (for $300 \mathrm{Myr}$; Burrows 2002, private communication). The area between the vertical dotted lines marks the locus of the inferred planet (Gorkavyi \& Taidakova 2001; Wilner et al. 2002). Thirteen per cent of the total area imaged has twice the integration time and hence $\sim 0.4$ mag better sensitivity, which is not accounted for in this analysis. The limiting magnitude along the brightest ray at P.A. $=50^{\circ}$ (see Fig. 1) is $\sim 1$ mag poorer (as realized in the uppermost points in the graph) than along directions with no bright artifacts. No limiting magnitudes are inferred for the $\sim 4^{\prime \prime} \times 4^{\prime \prime}$ area covered by the "ghost" in the north field.

from $\Delta H=12.5 \mathrm{mag}$ at $4^{\prime \prime}$ to $\Delta H=19 \mathrm{mag}$ at $\geq 26^{\prime \prime}$, 1.8 mag brighter than for low-background observations.

Extensive artificial star experiments were not performed for the less well-registered parts of the mosaic: the Vega $\mathrm{S}$ and Vega $\mathrm{W}$ fields. However, after applying the registration method used for these fields (ray intersection) to the Vega E field, for which centroiding provided the best registration among our fields, we observe that the faintest object in Vega $\mathrm{E}(H=18.9)$ is at the detection limit $(\mathrm{S} / \mathrm{N}=4.7)$. The detection limit is thus 0.3 mag brighter than the $H=19.2$ found at that location using centroiding. Since smearing of point sources due to improper registering is uniform across the image (there being only translational and no rotational degrees of freedom), we estimate limiting magnitudes in Vega $\mathrm{S}$ and $\mathrm{W}$ of $\sim 0.3$ mag brighter than in Vega $\mathrm{E}$ and $\mathrm{N}$.

\subsection{Comparisons to Models}

Figures 3 and 4 show the photometric measurements from Table 1 for the detected point sources assuming a common distance modulus with Vega, along with a 300 Myr isochrone for 1-30 $M_{\mathrm{J}}$ objects (Burrows et al. 2001) and known field $\mathrm{L}$ and $\mathrm{T}$ dwarfs (whose ages may range from $0.5-$ 10 Gyr). Given their colors, all sources detected by us in the vicinity of Vega are too red compared with the expected locus of planetary-mass companions (from Table 1) and too faint to be brown dwarfs. Hence, they are most likely back- ground stars. This was confirmed for objects $1-5$ by a relative proper motion test, with the positions of objects 2-5 (measured from PSF fits) compared to that of object 1 . None changed by more than $17 \pm 15$ mas $=0.68 \pm 0.60$ pixels between the two epochs. The proper motion for Vega over the period (67 days) was $64.4 \pm 0.8$ mas $=2.56 \pm 0.03$ pixels (Hipparcos), and hence any projected companion that is gravitationally bound to Vega should have moved by this amount (barring all five being associated).

No colors or proper motion information are available for source 6; hence, we can only estimate its likelihood of association with Vega from the expected frequency of field stars. To assess background contamination, we used the SKY model of Wainscoat et al. (1992), which for the position of Vega $(l=67.45, b=19.24)$ gives a $7.6^{\circ} \%$ probability that four or more stars of the specified magnitudes (for objects 1-3 and 6) are seen in the deep image. Thus, our detections are statistically consistent with being background stars.

Our results, nevertheless, demonstrate that detection of planetary-mass companions to nearby stars with groundbased telescopes is a real possibility. Based on their $H$ magnitudes, we list the predicted masses of the candidate companions in the last column of Table 1, assuming a common distance modulus with Vega and using models from Burrows (2002, private communication) and Chabrier et al. (2000) for a $300 \mathrm{Myr}$ old star. In using the Chabrier et al. models, linear interpolation has been applied between the values for $100 \mathrm{Myr}$ and $500 \mathrm{Myr}$. Both sets of models include internal heating processes only and not, e.g., irradiation of the planetary atmosphere by the star or reflected light from the star, but are appropriate given the large orbital separation of our candidate companions and the wavelength regime in which we are working. We should have detected any planets/brown dwarfs greater than $10 M_{\mathrm{J}}$ at separations greater than $12^{\prime \prime}(90 \mathrm{AU})$ and greater than $5 M_{\mathrm{J}}$ at greater than $20^{\prime \prime}$ (160 AU) from Vega.

\section{DISCUSSION}

Based on proper motion, colors, and field star considerations, it is unlikely that the newly discovered objects are substellar companions to Vega. Yet their existence in close proximity to Vega is heretofore unappreciated.

With respect to the predicted 2-3 $M_{\mathrm{J}}$ planetary perturber to Vega's dust distribution, Gorkavyi \& Taidakova (2001; see also Ozernoy et al. 2000) quote exact positions of a possible planet, with orbital radius $\sim 100$ AU. We find no $H<16.5$ objects ( $>8 M_{\mathrm{J}}$; Burrows et al. 2001 models) at either of their quoted positions or along the line connecting them, which may also be solutions to the model. Point sources are found neither along the Wilner et al. (2002) planetary orbit nor anywhere within the 14" submillimeter emission (albeit at lower sensitivity limits: $H<17-13$; $>7-30 M_{\mathrm{J}}$ ).

How do our upper limits compare to others in the literature for Vega? Gatewood \& de Jonge (1995) find no astrometric evidence for planets greater than $12 M_{\mathrm{J}}$ at 1.5-5.0 AU (1.2-7 yr period). Holland et al. (1998) place an upper limit of $12 M_{\mathrm{J}}$ on companions based on null result observations with Keck/NIRC, though no details are given. The NICMOS images of Silverstone et al. (2002) have similar sensitivity to ours (to within $0.5 \mathrm{mag}$ at 1.10 and $2.05 \mu \mathrm{m}$ ); however, they cover an area too small to see any of the objects detected by us. The Oppenheimer (1999) survey of stars within 8 pc, which just barely included Vega, found no 


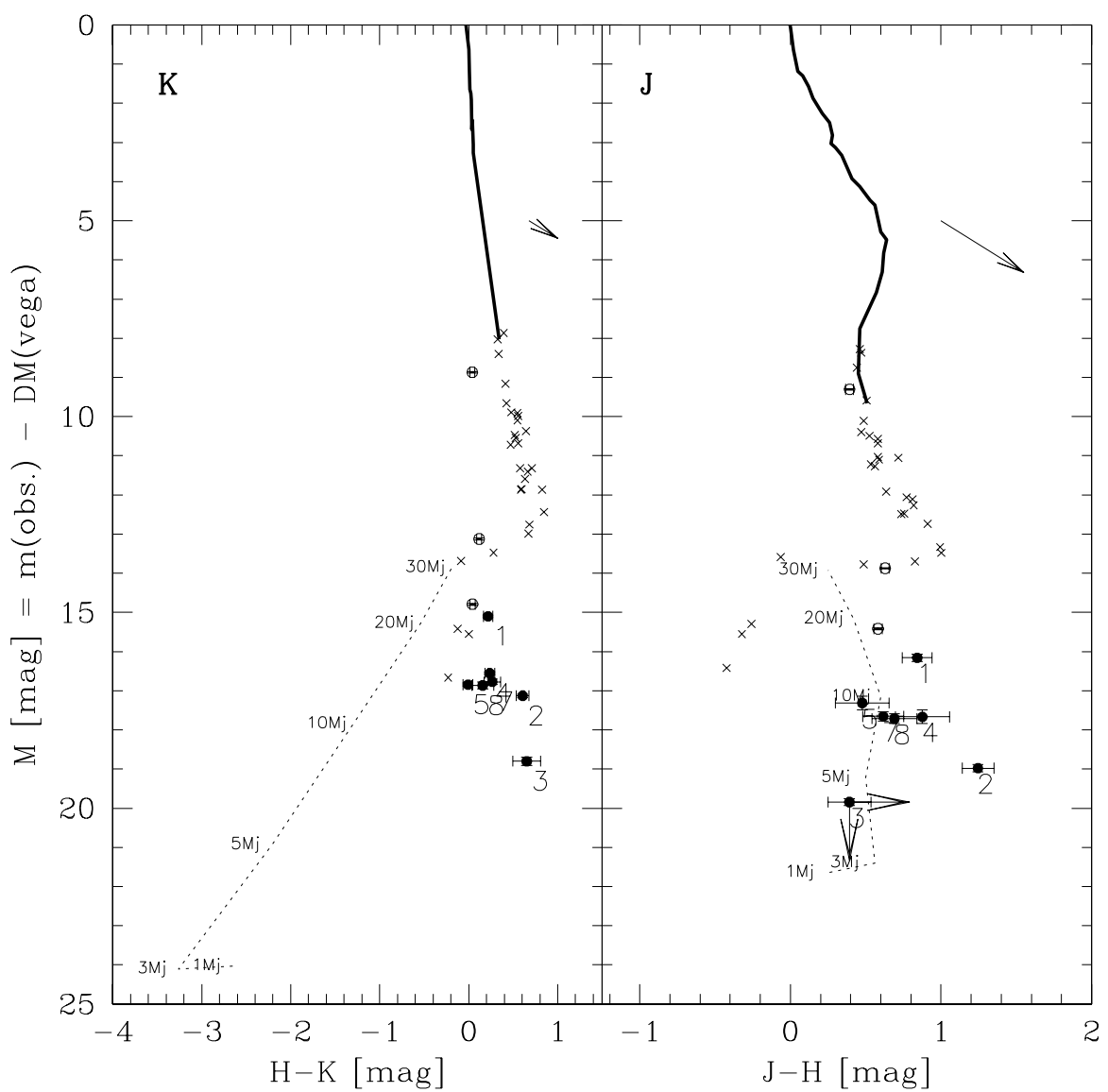

FIG. 3.- $J H K_{\mathrm{s}}$ color-magnitude diagrams in the CIT photometric system. The heavy solid line is the main- sequence relation for spectral types A0-M6 and the crosses are M4-T6 dwarfs from Leggett et al. (2002). The dotted line is the Burrows et al. (2001) 300 Myr isochrone for masses 1-30 $M_{\mathrm{J}}$, as labeled. The arrow corresponds to $5 \mathrm{mag}$ of interstellar reddening. Filled circles with error bars represent our Vega field data, while open circles are the calibration field data.

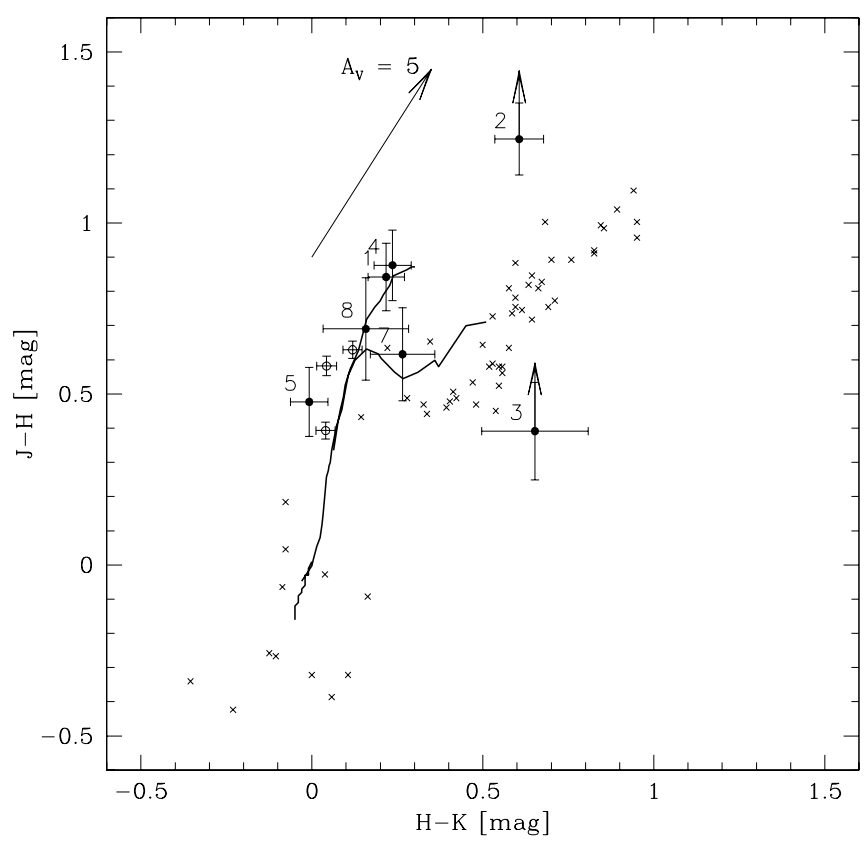

FIG. 4.-JHK color-color diagram in the CIT photometric system. See Fig. 3 for a description of the symbols. companions at the positions of our detections. Based on their sensitivity curves, objects brighter than $r=16-17 \mathrm{mag}$ should have been detected around Vega from 20"-30". Given the $R-H$ colors of low-mass stars for which $H-K=0.1-0.3$ (K2-M5 spectral types: $R-H>2.0$ ), the Oppenheimer survey may have just missed detecting our brightest projected companion to Vega if it is a background star as early as $\mathrm{K} 2$. Our spectrum $(\mathrm{S} / \mathrm{N} \approx 15)$ of object 1 indeed places it in the $\mathrm{K} 5 \mathrm{~V}-\mathrm{M} 5 \mathrm{~V}$ spectral type range.

Our imaging data can also be used to test a possible cosmological origin of the submillimeter dust clumps around Vega. Spectral energy distributions of (sub-) millimeter galaxies (Dannerbauer et al. 2002; Goldader et al. 2002; Klaas et al. 2001) suggest $z \gtrsim 1$ for any responsible background galaxy, given our nondetection at $H$ band. However, deep searches for $K$-band counterparts to several submillimeter galaxies have reached $K \approx 22 \mathrm{mag}$ (e.g., Dannerbauer et al. 2002) with no counterpart detection, suggesting that our data may be too insensitive (by 6-7 mag at these locations) to put a sensible limit on this hypothesis.

\section{CONCLUSIONS}

We find eight faint objects within $35^{\prime \prime}$ of Vega that are 15 19 mag fainter than the star at $H$ band. If associated, at the 330 Myr age for Vega, current brown dwarf cooling models 
(Burrows et al. 2001; Chabrier et al. 2000) set their masses at 5-35 $M_{\mathrm{J}}$. The number of detected objects is however consistent with estimates of field star density, and their colors and proper motion indicate that they are not associated with Vega.

We thus exclude the possibility of a distant (80-220 AU; $\sim 83 \%$ of this area is imaged), massive $\left(>10 M_{\mathrm{J}}\right.$; $>6 M_{\mathrm{J}}$ for 120-220 AU) planetary/brown dwarf companion causing the observed dust distribution around Vega. We also detect nothing at the positions of the predicted planetary perturbers, with upper mass limits of $7-15 M_{\mathrm{J}}(H<17-13)$, well above the $2-3 M_{\mathrm{J}}$ predictions. We detect nothing at the position of the mid-infrared dust clumps, placing limits on the possibility of their extragalactic interpretation.
We acknowledge with appreciation those who have endeavored over the years to further the development of the adaptive optics system at Palomar. In particular, we have benefited substantially from conversations with T. Hayward, M. Troy, R. Dekany, and R. Burruss, and enjoyed the expert assistance at the telescope of J. Mueller and K. Dunscombe. J. Carpenter also participated in some of the data acquisition. This publication makes use of data products from the Two Micron All Sky Survey, which is a joint project of the University of Massachusetts and the Infrared Processing and Analysis Center, California Institute of Technology, funded by the National Aeronautics and Space Administration and the National Science Foundation.
Aumann, H. H., et al. 1984, ApJ, 278, L23

Backman, D. E., \& Paresce, F. 1993, in Protostars and Planets III, ed. E. H. Levy \& J. I. Lunine (Tucson: Univ. Arizona Press), 1023

Beust, H., Lagrange-Henri, A. M., Vidal-Majar, A., \& Ferlet, R. 1989, A\&A, 223, 304

Beust, H., Vidal-Majar, A., Ferlet, R., \& Lagrange-Henri, A. M. 1990, A\&A, 236, 202

Bloemhof, E. E., Marsh, K. A., Dekany, R. G., Troy, M., Marshall, J., Oppenheimer, B. R., Hayward, T. L., \& Brandl, B. 2000, Proc. SPIE, 4007, 889

Burrows, A., Hubbard, W. B., Lunine, J. I., \& Liebert, J. 2001, Rev. Mod. Phys., 23, 719

Burrows, A., et al. 1997, ApJ, 491, 856

Chabrier, G. Baraffe, I., Allard, F., \& Hauschildt, P. 2000, ApJ, 542, 464

Ciardi, D. R., van Belle, G. T., Akeson, R. L., Thompson, R. R., Lada, E. A., \& Howell, S. B. 2001, ApJ, 559, 1147

Dannerbauer, H., Lehnert, M. D., Lutz, D., Tacconi, L., Bertoldi, F., Carilli, C., Genzel, R., \& Menten, K. 2002, ApJ, 573, 473

Dent, W. R. F., Walker, H. J., Holland, W. S., \& Greaves, J. S. 2000, MNRAS, 314, 702

Gatewood, G., \& de Jonge, J. K. 1995, ApJ, 450, 364

Goldader, J. D., Meurer, G., Heckman, T. M., Seibert, M., Sanders, D. B., Calzetti, D., \& Steidel, C. C. 2002, ApJ, 568, 651

Gorkavyi, N. N., \& Taidakova, T. A. 2001, BAAS, 199, 86.07

Gulliver, A. F., Hill, G., \& Adelman, S. J. 1994, ApJ, 429, L81

Harper, D. A., Loewenstein, R. F., \& Davidson, J. A. 1984, ApJ, 285, 808

Harvey, P. M., Wilking, B. A., \& Joy, M. 1984, Nature, 307, 441

\section{EFERENCES}

Hayward, T. L., Brandl, B., Pirger, B., Blacken, C., Gull, G. E., Schoenwald, J., \& Houck, J. R. 2001, PASP, 113, 105

Heinrichsen, I., Walker, H. J., \& Klaas, U. 1998, MNRAS, 293, L78

Holland, W. S., et al. 1998, Nature, 392, 788

Kalas, P., \& Jewitt, D. 1996, AJ, 111, 1347

Klaas, U., et al. 2001, A\&A, 379, 823

Koerner, D. W., Sargent, A. I., \& Ostroff, N. A. 2001, ApJ, 560, L181

Kuchner, M. J., Brown, M. E., \& Koresko, C. D. 1998, PASP, 110, 1336

Leggett, S. K., et al. 2002, ApJ, 564, 452

Mannings, V., \& Barlow, M. J. 1998, ApJ, 497, 330

Oppenheimer, B. R. 1999, Ph.D. thesis, Caltech

Ozernoy, L. M., Gorkavyi, N. N., Mather, J. C., \& Taidakova, T. A. 2000, ApJ, 537, L147

Silverstone, M. D. 2000, Ph.D. thesis, Univ. of California at Los Angeles

Silverstone, M. D., Schneider, G., \& Smith, B. A. 2002, AAS Meeting, 200, 74.07

Smith, B. A., Fountain, J. W., \& Terrile, R. J. 1992, A\&A, 261, 499

Song, I., Caillault, J.-P., Barrado y Navascues, D., \& Stauffer, J. R. 2001, ApJ, 546, 352

Troy, M., et al. 2000, Proc. SPIE, 4007, 31

Wainscoat, R. J., Cohen, M., Volk, K., Walker, H. J., \& Schwartz, D. E. 1992, ApJS, 83, 111

Weissman, P. R. 1984, Science, 224, 987

Wilner, D. J., Holman, M. J., Kuchner, M. J., \& Ho, P. T. P. 2002, ApJ, 569, L115

Zuckerman, B., \& Becklin, E. E. 1993, ApJ, 414, 793 